\title{
Adiabatic and nonadiabatic behavior of the Carr-Purcell-Meiboom-Gill sequence in time-dependent magnetic fields
}

\author{
Martin D. Hürlimann $\odot,{ }^{*}$ Shin Utsuzawa $\odot,^{\dagger}$ and Chang-Yu Hou $\odot^{\ddagger}$ \\ Schlumberger-Doll Research, Cambridge, Massachusetts 02139, USA
}

(Received 6 March 2020; revised 4 June 2020; accepted 4 August 2020; published 2 September 2020)

\begin{abstract}
We investigate experimentally the effect of time-dependent magnetic fields on the spin dynamics of the CarrPurcell-Meiboom-Gill sequence. The measurements demonstrate that the evolution is controlled by an effective Hamiltonian that is a function of the offset field and the sequence parameters. Over a wide range of offset fields, ramp rates, and sequence parameters, the system follows the effective Hamiltonian adiabatically. In this regime, the echo amplitudes exhibit characteristic modulations that reflect the changing directions of the eigenvector associated with the zero-energy level. The experimental results are in excellent quantitative agreement with theoretical predictions. Nonadiabatic events are observed at distinct offsets where the system becomes nearly degenerate. These nonadiabatic events occur precisely at the field offsets predicted by theory. After passing through such nonadiabatic regions, an abrupt change in the occupation of the zero-energy level is observed. In the adiabatic regime, the effects of field fluctuations are demonstrated to be fully reversible, while the occurrence of nonadiabatic events leads to hysteresis. The location and range of the adiabatic regions can be manipulated by modifying the pulse parameters.
\end{abstract}

DOI: 10.1103/PhysRevResearch.2.033352

\section{INTRODUCTION}

When a quantum system is driven through multiple avoided energy-level crossings, the occurrence of nonadiabatic transitions in the vicinity of these crossings can lead to interesting quantum interference phenomena. A well-known example is the so-called Landau-Zener-Stückelberg interferometry technique, where a two-level quantum system is made to repeatedly traverse avoided crossings and to undergo LandauZener transitions [1]. The resulting constructive or destructive quantum interference pattern, sensitive to the amplitude and frequency of the driving field and the gap between the two levels, has been used to demonstrate and simulate such quantum phenomena as the universal conductance fluctuation and the weak localization [2,3].

This interference effect associated with nonadiabatic transitions at the passage near avoided crossings has been observed mostly in effective two-level systems with Rydberg atoms [4] and superconducting qubits [5,6]. Hence, alternative platforms to probe such interference phenomena are of interest, in particular when the underlying effective Hamiltonian can be readily modified or engineered. In our recent work [7], we demonstrated theoretically that the magnetization of

\footnotetext{
*mhurlimann@gmail.com

†SUtsuzawa@slb.com

${ }^{\ddagger}$ CHou2@slb.com
}

Published by the American Physical Society under the terms of the Creative Commons Attribution 4.0 International license. Further distribution of this work must maintain attribution to the author(s) and the published article's title, journal citation, and DOI. noninteracting nuclear spins $1 / 2$, subjected to a long string of repeating $\mathrm{rf}$ pulses and a temporally varying magnetic field, shows similar interference effects. In the fast pulsing regime, the observed magnetization is governed by a time-dependent Hamiltonian that has the form of a Zeeman interaction with an effective field $\vec{B}_{\text {eff }}$. The strength and direction of $\vec{B}_{\text {eff }}$ can be modified by the choice of experimental parameters of the pulse sequence, such as the repetition rate and durations of the pulses. Here we present experimental confirmations of the occurrences of adiabatic passages and nonadiabatic transitions in this system as predicted by the effective Hamiltonian theory. The measurements were performed using standard nuclear magnetic resonance (NMR) techniques with the addition of a controlled linear ramping of the external magnetic field.

The pulse sequence used in our experiments consists of an initial excitation pulse followed by a long string of refocusing pulses, separated by the echo spacing $t_{E}$. The magnetization is probed stroboscopically at the nominal echo times $k t_{E}$, where $k$ is a positive integer. This sequence is well known in standard NMR applications where it is referred to as the Carr-Purcell-Meiboom-Gill (CPMG) sequence [8,9]. It is commonly used to probe dynamic processes $[10,11]$, to enhance the signal [12], or to reduce decoherence due to external noise or diffusion effects [13-15]. When used in quantum measurement protocols, the technique is usually referred to as dynamic decoupling [13]. This sequence also underlies essentially all applications in grossly inhomogeneous magnetic fields $[16,17]$. While the sequence was originally designed for operation in a static external magnetic field, here we study the response in the presence of a time-varying external field. The main motivation is to explore new physics, but this investigation also benefits the understanding of conventional measurements in unstable fields or when there is relative 
motion between the sample and measurement apparatus. We consider here time-dependent external fields that can vary during the CPMG sequence by an amount comparable to or larger than the strength of the rf field.

The paper is organized as follows. The theoretical treatment is reviewed in Sec. II. Section III presents the experimental setup and Sec. IV presents the measurements and main results. We demonstrate that the response is well described by the effective Hamiltonian approach presented in [7]. The experimental results can be characterized by regions of adiabatic behavior that are interrupted by nonadiabatic events. The summary in Sec. V discusses the implication of these results.

\section{THEORETICAL CONSIDERATIONS}

The theoretical analysis in [7] is based on the inspection of a single refocusing cycle, in which the evolution of the magnetization for a complete cycle is defined by an unitary operator governed by the effective Hamiltonian

$$
H_{\mathrm{eff}}=\hbar \gamma \vec{B}_{\mathrm{eff}} \cdot \overrightarrow{\mathcal{S}}=\hbar \frac{\alpha\left(\omega_{0} ; \omega_{1}, t_{E}, t_{p}\right)}{t_{E}} \hat{n}\left(\omega_{0} ; \omega_{1}, t_{E}, t_{p}\right) \cdot \overrightarrow{\mathcal{S}}
$$

Here $\overrightarrow{\mathcal{S}}=\left(\mathcal{S}_{1}, \mathcal{S}_{2}, \mathcal{S}_{3}\right)$ are the generators of rotations with respect to the three axes in the Cartesian coordinate system with their matrix elements given by $\left(\mathcal{S}_{k}\right)_{i j}=-i \epsilon_{i j k}$. In addition, $\vec{B}_{\text {eff }}$ is the effective magnetic field that characterizes the average Hamiltonian of a refocusing cycle and $\gamma$ is the gyromagnetic ratio. The magnitude of $\vec{B}_{\text {eff }}$ is proportional to the phase $\alpha$ and its direction is given by the unit vector direction $\hat{n}$. The quantities $\alpha$ and $\hat{n}$ (and therefore the effective Hamiltonian) depend on the offset between the Larmor and $\mathrm{rf}$ frequencies $\omega_{0} \equiv \gamma B_{0}-\omega_{r f}$, the nutation frequency $\omega_{1} \equiv \gamma B_{1, \perp} / 2$, the echo spacing $t_{E}$, and the duration of the pulses $t_{p}$. Explicit forms of these dependences are given in Appendix A. It is worth noting that the effective Hamiltonian can be further modified by replacing the single rf pulses by composite or frequency modulated pulses [18].

Now the most economical way to analyze the echo-to-echo propagation of the magnetization is to decompose it into its eigenmodes. Critically, the effective Hamiltonian in Eq. (1) has a zero-energy eigenmode with the corresponding eigenvector $\hat{n}$. We will refer to this mode as the CPMG mode with its corresponding unitary evolution having unity eigenvalue. The initial excitation pulse of the CPMG pulse sequence is designed to populate this particular eigenmode. Magnetization of this eigenmode is consistently refocused from echo to echo, even in the presence of field inhomogeneities. The evolution of this mode is characterized by the absence of a dynamic and geometric phase. In contrast, the other eigenmodes [usually referred to as Carr-Purcell (CP) modes] for the unitary evolution have eigenvalues of the form $e^{ \pm i \alpha}$ that vary with $\omega_{0}$. In the presence of spatial field inhomogeneities across the sample, this dispersion gives rise to a rapid echo-to-echo dephasing of the CP magnetization component, in analogy to common $T_{2}^{*}$ dephasing of the free-induction decay. Therefore, the detected signal is typically completely dominated by the CPMG component.
With a time-dependent external field $B_{0}(t)$, it was shown in [7] that in the fast pulsing regime, the evolution of the magnetization from echo to echo is again governed by a time-dependent effective Hamiltonian. To the lowest order, the time-dependent effective Hamiltonian closely follows the form of Eq. (1) and can be approximated as

$$
H_{\mathrm{eff}}=\hbar \gamma \vec{B}_{\mathrm{eff}}(t) \cdot \overrightarrow{\mathcal{S}} \approx \hbar \frac{\alpha\left(\omega_{0}(t)\right)}{t_{E}} \hat{n}\left(\omega_{0}(t)\right) \cdot \overrightarrow{\mathcal{S}} .
$$

When the external field is changed sufficiently slowly, we expect that the magnetization follows the evolving eigenmodes adiabatically. The magnetization of the CPMG components is effectively spin locked to its eigenvector, as shown in Eq. (B1). On resonance, this eigenvector lies in the transverse plane, but it acquires a longitudinal component as the amplitude of the external field is increased. This change in direction is a nonmonotonic function of $\omega_{0}$ and results in a systematic modulation of the detected signal as the applied field is changed. In the adiabatic regime, the change of signal induced by a fluctuating external field is reversible and independent of the path of the fluctuation.

To stay in this adiabatic regime, the adiabatic condition $\mathcal{A} \gg 1$ has to be fulfilled. As discussed in detail in [7], the adiabaticity parameter $\mathcal{A} \equiv v_{0, \text { crit }} /\left(d \tilde{\omega}_{0} / d \tau\right)$ is the ratio of the instantaneous critical velocity $v_{0, \text { crit }}$, an intrinsic parameter, and the dimensionless ramp rate of the external field $d \tilde{\omega}_{0} / d \tau \equiv d \frac{\omega_{0}(t)}{\omega_{1}} / d \frac{t}{t_{E}}$, an experimental parameter. The critical velocity $v_{0, \text { crit }}$ is controlled by the change of the direction $\theta$ of the eigenvector $\hat{n}$ with offset frequency $\omega_{0}$ and the strength of the effective field $\alpha / t_{E}=\gamma\left|\vec{B}_{\text {eff }}\right|: v_{0, \text { crit }} \equiv \alpha /\left(d \theta / d \tilde{\omega}_{0}\right)$.

At distinct offset frequencies where the modes become nearly degenerate, $\alpha \approx 0$ [cf. Fig. 7(a)] for $\alpha$ as a function of $\omega_{0} / \omega_{1}$, the critical velocity $\nu_{0, \text { crit }}$ has pronounced minima. When the variable external field reaches a value corresponding to one of these minima, the spin dynamics will likely become nonadiabatic, except when the ramp rate becomes very small. Transitions between the CPMG and CP modes then occur with the passage through these points, similar to the Landau-Zener transition for traversing an avoided level crossing. Following the theoretical consideration in [7], the temporal interferences are also expected to occur when multiple near-degenerate points are traversed. The direct observation of the interference between the CPMG and $\mathrm{CP}$ modes in the resulting macroscopic signal requires that the time-dependent magnetic field has a very high spatial uniformity across the sample. In our setup, the applied field exhibits some residual spatial inhomogeneity that leads to a spatial averaging out of the net $\mathrm{CP}$ magnetization after the transition. This prevents a direct observation of the interference effect in our experiments. However, we can detect abrupt changes in the occupation of the CPMG level. Such abrupt changes are a clear sign of the occurrence of nonadiabatic transitions that generate the interference effects on the microscopic level. The refocusing of the $\mathrm{CP}$ mode at specific detuning in our experiments (cf. Appendix $\mathrm{C}$ ) demonstrates that the local $\mathrm{CP}$ magnetization has a long intrinsic coherence time. This indicates that the direct observation of interference effect becomes possible if the spatial averaging effect can be avoided by either improvement of the field homogeneity or the miniaturization of the sample. 


\section{EXPERIMENTAL SETUP}

Experiments were performed on a cylindrically shaped water sample (11 $\mathrm{mm}$ diameter, $10 \mathrm{~mm}$ length) that was inserted in a solenoid $\mathrm{rf}$ coil and placed in a horizontal superconducting imaging magnet (Nalorac) with a $30-\mathrm{cm}$ bore. The rf was set to $85.1 \mathrm{MHz}$, the Larmor frequency of the magnet. The rf power was adjusted so that the duration of the nominal $180^{\circ}$ pulse resulted in $t_{180}=40 \mu \mathrm{s}$. This corresponds to a nutation frequency $\frac{\omega_{1}}{2 \pi}=\frac{1}{2 t_{180}}=12.5 \mathrm{kHz}$.

We applied the standard CPMG sequence to the rf coil consisting of an initial $90^{\circ}$ pulse followed by a long train of up to $8956180^{\circ}$ pulses. Standard two-step phase cycling was used. The echo spacing $t_{E}$ was set at values between $6.4 t_{180}$ and $24 t_{180}$ as indicated. The complex echo amplitudes were acquired at the nominal echo locations halfway between the refocusing pulses.

During the measurements, the $B_{0}$ field applied to the sample was varied by up to $1.29 \mathrm{mT}$, corresponding to a change in Larmor frequency $\Delta \omega_{0}$ of $4.4 \omega_{1}$. This was accomplished using a commercial gradient set (Bruker BGA12SL) that was physically offset by $8 \mathrm{~cm}$ along its axis. At the site of the sample, this arrangement generated an essentially uniform bias field (rather than a gradient field in the center of the gradient set). The strength and time dependence of this bias field was controlled through the spectrometer by adjusting the current through the gradient setup. The spatial uniformity of the bias field across the sample can be approximated by a Gaussian distribution with a relative width of $6.7 \times 10^{-3}$. The bias field was typically ramped up linearly from zero to a maximum value with a constant ramp rate. Measurements were also performed with a linear ramp-up immediately followed by a linear ramp-down. We use the dimensionless quantity $d \tilde{\omega}_{0} / d \tau \equiv d \frac{\omega_{0}(t)}{\omega_{1}} / d \frac{t}{t_{E}}$ to characterize the ramp rate. This normalized ramp rate measures the change of the Larmor frequency during one refocusing cycle relative to the rf nutation frequency $\omega_{1}$ (which is proportional to the excitation bandwidth). We present results for $d \tilde{\omega}_{0} / d \tau$ between $5 \times 10^{-4}$ and $8 \times 10^{-3}$.

It is useful to relate these ramp rates to possible causes of field fluctuations, in particular to magnet drifts and sample displacements in a gradient $B_{0}$ field. With the quoted experimental parameters of rf power and echo spacings, the range of $d \tilde{\omega}_{0} / d \tau$ corresponds to relative magnet drift rates $\frac{1}{\omega_{r f}} \frac{d \omega_{0}(t)}{d t}=$ $\frac{\omega_{1}^{2}}{\pi \omega_{r f}} \frac{t_{180}}{t_{E}} \frac{d \tilde{\omega}_{0}}{d \tau}$ between 77 and $4590 \mathrm{ppm} / \mathrm{s}$. Alternatively, when the $B_{0}$ fluctuations are caused by sample motion in a gradient $g$, the normalized ramp rate $d \tilde{\omega}_{0} / d \tau$ is associated with motion $\frac{d x}{d t}=\frac{\omega_{1}^{2}}{\pi \gamma g} \frac{t_{180}}{t_{E}} \frac{d \tilde{\omega}_{0}}{d \tau}$. For a gradient $g=20 \mathrm{mT} / \mathrm{m}$ found in a typical magnetic resonance imaging setup, the range of our normalized ramp rates corresponds to a range of $\frac{d x}{d t}$ of $8-460 \mathrm{~mm} / \mathrm{s}$.

For each measurement of echo amplitudes $M(t)$ with a time-dependent bias field, an auxiliary measurement $M_{\text {aux }}(t)$ without a bias field but with otherwise identical parameters was performed. The auxiliary measurement was used to phase both sets of data. The phased echo amplitudes $M(t)$ were then normalized with respect to the auxiliary measurements to compensate for relaxation effects. We present results in terms of the normalized signal $S(t) \equiv M(t) / M_{\text {aux }}(t)$.
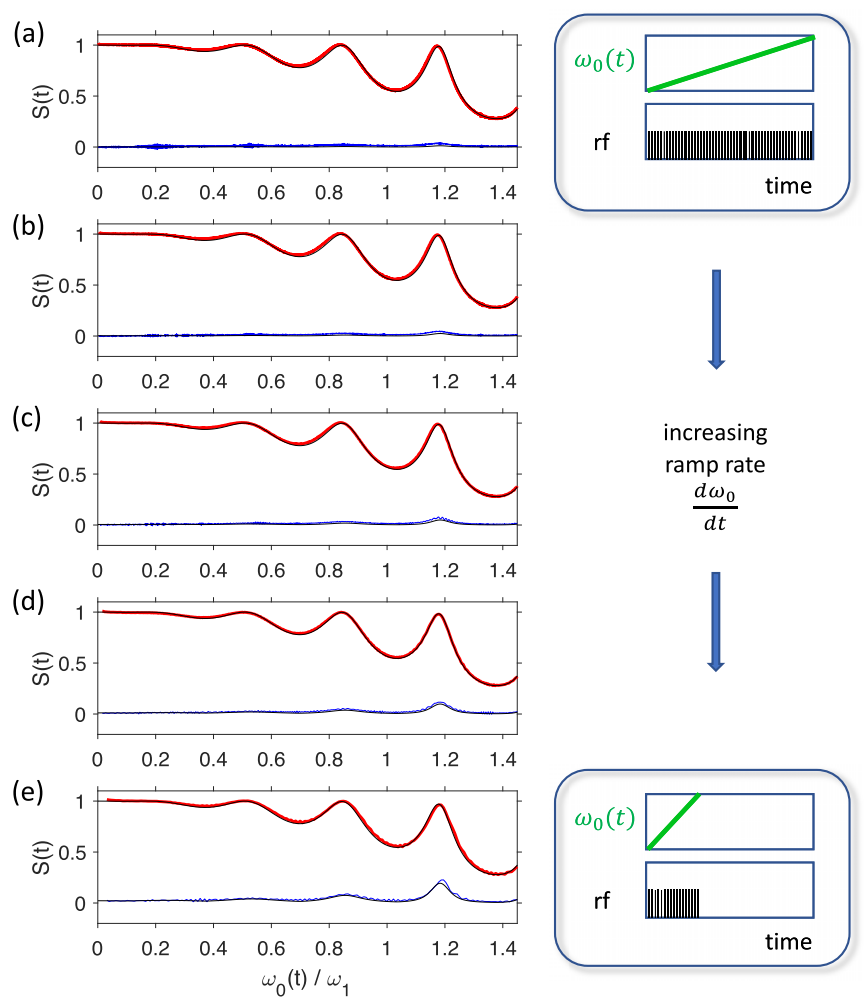

FIG. 1. Normalized in-phase (red) and out-of-phase (blue) echo amplitudes $S(t)$ versus the instantaneous offset in normalized Larmor frequency, $\omega_{0} / \omega_{1}$, for a CPMG sequence with a linearly increasing $B_{0}$ field starting on resonance. The ramp rates $d \tilde{\omega}_{0} / d \tau$ are (a) $5 \times$ $10^{-4}$, (b) $1 \times 10^{-3}$, (c) $2 \times 10^{-3}$, (d) $4 \times 10^{-3}$, and (e) $8 \times 10^{-3}$. The echo spacing was fixed at $t_{E}=6.4 t_{180}$. The experimental measurements are well described by the analytical results for adiabatic dynamics given by Eqs. (B2), shown as black lines. The panels on the right give a schematic indication of the experimental procedure. Note that the actual pulse spacings are much smaller and the total number of pulses is much larger than could be drawn.

\section{RESULTS}

\section{A. Moderate field variations: Adiabatic behavior}

We first consider the case of a linear field ramp that starts from resonance. We display the relaxation compensated amplitudes for echoes forming at time $t, S(t)$, versus the normalized offset of Larmor frequency $\tilde{\omega}_{0}(t)=\omega_{0}(t) / \omega_{1}$ at that time. In these graphs, the individual echoes are separated by $d \tilde{\omega}_{0} / d \tau$ along the abscissa. Since the normalized ramp rates are always much smaller than 1 , the graphs of the discrete points of the individual echo amplitudes appear as solid lines.

In the experiments shown in Fig. 1, five different normalized ramp rates $d \tilde{\omega}_{0} / d \tau$ between $5 \times 10^{-4}$ and $8 \times 10^{-3}$ were used to increase the offset frequency $\omega_{0}$ from zero (i.e., resonance) to $1.45 \omega_{1}$. The signals show systematic modulations as a function of the offset frequency. The echo amplitudes are predominantly in phase with the refocusing pulses and display only a weak dependence on the ramp rate.

These results are consistent with the expected behavior in the adiabatic regime. The $90^{\circ}$ excitation pulse generates transverse magnetization in phase with the refocusing pulses. In the static on-resonance case, this initial magnetization is an 
eigenstate of the propagator with unity eigenvalue, i.e., the socalled CPMG subspace. In time-dependent fields, it remains approximately an eigenstate on resonance as long as $\delta \epsilon \equiv$ $\frac{t_{E}^{2}}{8} \frac{d \omega_{0}}{d t} \ll 1$. In our experiments, $\delta \epsilon$ lies between $1.3 \times 10^{-3}$ and $2.0 \times 10^{-2}$ and fulfills this condition well. (For larger echo spacings, the initial magnetization has both CPMG and $\mathrm{CP}$ components resulting in the well-known even-odd echo modulation [8].)

As the applied field is increased, the initial magnetization remains in the CPMG eigenspace and follows the field variations adiabatically if the ramp rate is smaller than the offset-dependent critical velocity. The minimum critical velocity in the range of offset frequencies up to $1.45 \omega_{1}$ is $v_{0, \text { crit }}=0.042$, i.e., more than 5 times larger than the highest ramp rate $d \tilde{\omega}_{0} / d \tau$ used. Therefore, the adiabatic condition $\mathcal{A} \gg 1$ is fulfilled and the magnetization is effectively spin locked to the CPMG eigenvector. The observed signal modulation reflects the variable direction of the eigenvector with offset frequency $\omega_{0}$.

The CPMG eigenvector of the propagator has no out-ofphase component in the stationary case and its transverse component is given by Eq. (A1). For finite ramp rates, there are slight modifications that can be expanded in terms of $1 / \mathcal{A}$. The first-order results are given by Eqs. (B2) and show some small out-of-phase components proportional to $1 / \mathcal{A}$. For a comparison with the experimental results in Fig. 1, we have convoluted the theoretical expressions in Eqs. (B2) with the known relative field inhomogeneity of $6.7 \times 10^{-3}$ of the applied offset field $\omega_{0}$. The experimental results are in excellent agreement with this prediction for full occupation of the CPMG mode, i.e., $a_{C P M G}=1$. This demonstrates that the modulation of the observed echo magnetization is controlled by the changing direction of the CPMG eigenvector.

As a further test, we have measured the response for different echo spacings over the same range of offset frequencies. Here we kept the normalized ramp rate fixed at $5 \times 10^{-4}$. The results are presented in Fig. 2.

The adiabaticity condition $\mathcal{A} \gg 1$ is again fulfilled for this range of experimental parameters, and we expect adiabatic behavior. The experiments show a more pronounced signal modulation for the measurements with longer echo spacings. This is in quantitative agreement with the expectations for the adiabatic regime for all echo spacings, based on Eqs. (A1) and (B2).

\section{B. Larger field variations: Observation of nonadiabatic events}

When the field ramp extends over a larger range of offset frequencies, deviations from the adiabatic behavior are observed even for moderate ramp rates. This is apparent from the results presented in Fig. 3, which shows the in-phase signal for a linear field ramp starting from 0 up to an offset frequency of $4.4 \omega_{1}$ at a ramp rate of $5 \times 10^{-4}$ for three different echo spacings. For small to moderate frequency offsets, the experimental results follow the theoretical expectation for the adiabatic regime as discussed above with $a_{C P M G}=1$.

However, beyond a distinct offset frequency, the experimental results abruptly start to deviate from the adiabatic predictions with $a_{C P M G}=1$. These abrupt changes occur
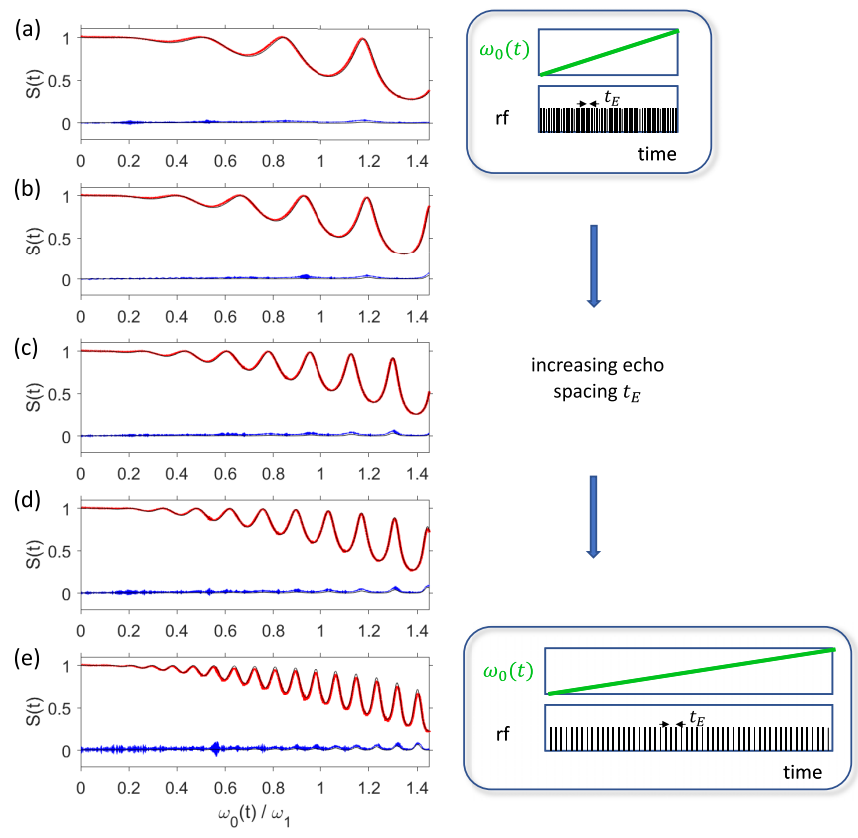

FIG. 2. Measured in-phase (red) and out-of-phase (blue) echo amplitudes for a CPMG sequence with a linearly increasing $B_{0}$ field, starting on resonance for different echo spacings. The normalized echo spacings $t_{E} / t_{180}$ are (a) 6.4 , (b) 8.0 , (c) 12.0 , (d) 15.0 , and (e) 24.0. The normalized ramp rate $d \tilde{\omega}_{0} / d \tau$ was fixed at $5 \times 10^{-4}$. The signal was normalized by a standard CPMG signal to account for relaxation. The black lines show the analytical results for adiabatic dynamics given by Eqs. (B2). The panels on the right give a schematic indication of the experimental procedure.

precisely at offset frequencies where the adiabaticity parameter $\mathcal{A}$ drops towards 1 or lower, as indicated in Fig. 3 in grayscale. At these offset frequencies, the critical velocity
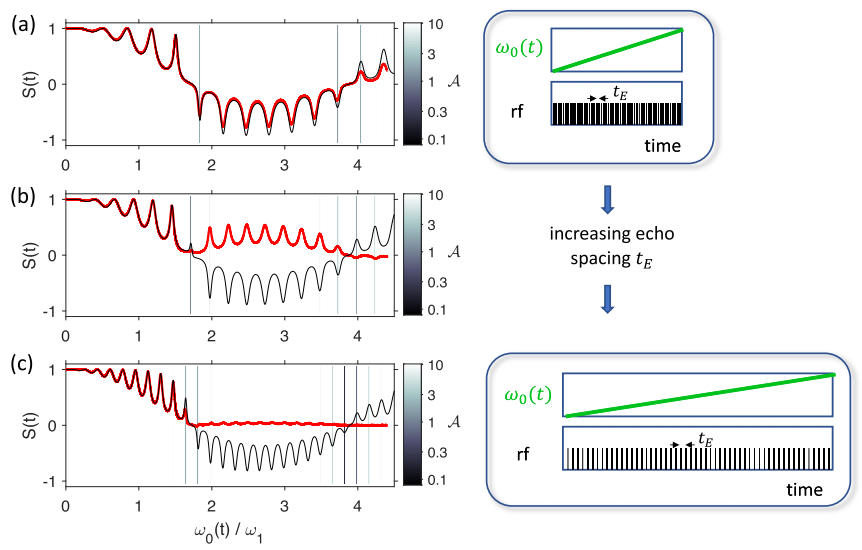

FIG. 3. Measured in-phase (red) echo amplitudes for a CPMG sequence with a linearly increasing $B_{0}$ field, starting on resonance for different echo spacings. The signal was normalized by a standard CPMG signal to account for relaxation. The normalized ramp rate $d \tilde{\omega}_{0} / d \tau$ was fixed at $5 \times 10^{-4}$. The normalized echo spacings $t_{E} / t_{180}$ are (a) 6.4 , (b) 8.0, and (c) 12.0. The black lines show the lowestorder analytical result, Eq. (B1) for $a_{C P M G}=1$. The calculated values of $\mathcal{A}$ are displayed in grayscale. The panels on the right give a schematic indication of the experimental procedure. 
$v_{0, \text { crit }}\left(\omega_{0}\right)$ has pronounced minima and the adiabatic condition $\mathcal{A} \gg 1$ is not fulfilled anymore. This leads to nonadiabatic transitions between the CPMG and the CP eigenspace.

During the crossing of these nonadiabatic regions, magnetization is exchanged between the CPMG and CP subspaces. The dispersion of the eigenvalues of the $\mathrm{CP}$ mode leads to a quick dephasing of the magnetization associated with the $\mathrm{CP}$ subspace in the presence of the slight inhomogeneity in the applied bias field (in analogy to the $T_{2}^{*}$ process). The detected signal is therefore dominated by the remaining magnetization of the dispersionless CPMG mode. After a nonadiabatic region of field offset is passed, the spin dynamics enters again an adiabatic region. The signal follows closely a rescaled version of the adiabatic expression (B1), with a scaling factor $a_{C P M G}$ less than 1 that indicates a reduced occupation of this mode. As is evident from Fig. 3, the amplitudes $a_{C P M G}$ can be either positive or negative.

As the external field is further increased, additional nonadiabatic regions are encountered that further modify the modal amplitude $a_{C P M G}$. The individual nonadiabatic regions are well separated and narrow with respect to the normalized offset frequency $\omega_{0} / \omega_{1}$. The results of Fig. 3 demonstrate that the adiabaticity parameter $\mathcal{A}$ is an accurate indicator for the location of the nonadiabatic regions.

It is challenging to predict the change in amplitude of the CPMG mode $a_{C P M G}$ after passing a nonadiabatic region. The width of these regions is narrow with respect to $\omega_{0} / \omega_{1}$, but generally much wider than the normalized ramp rate. Therefore, the traversing of a particular nonadiabatic region occurs over many refocusing cycles. The net change of the CPMG modal amplitude is the result of the accumulated transition rates between the CPMG and CP subspaces over all these cycles. As was shown in [7], these net rates are sensitive to the experimental parameters and they are affected by the field inhomogeneities. While the location of this nonadiabatic event can be accurately predicted, the associated changes in amplitude are therefore more difficult to predict robustly.

\section{Reversibility}

Further insight can be gained from experiments performed with bilinear field ramps shown in Fig. 4. The field was linearly ramped up from on resonance to a maximum value followed by a ramp-down back to on resonance. The magnitude of the ramp rates $\left|d \tilde{\omega}_{0} / d \tau\right|$ during the field ramp-up and -down was fixed at $10^{-3}$. The measurements were repeated with systematically varied maximum field values $\Delta B_{0}$ that correspond to frequency offsets $\Delta \omega_{0} \equiv \gamma \Delta B_{0}$.

The results show an interesting dependence on $\Delta \omega_{0}$. When $\Delta \omega_{0}$ is smaller than the first nonadiabatic region [Figs. 4(a) and 4(b)], the responses of normalized echo amplitudes versus instantaneous offset frequency during the ramp-up and ramp-down are identical. When the field is returned to its original value, the spin echoes are fully refocused (up to unavoidable relaxation). This reversibility indicates a purely adiabatic behavior with unity eigenvalue for the CPMG mode. In this regime, the evolution caused by the field fluctuation is fully reversible, including the dephasing caused by the inhomogeneities of the bias field. Note that this is even the case for

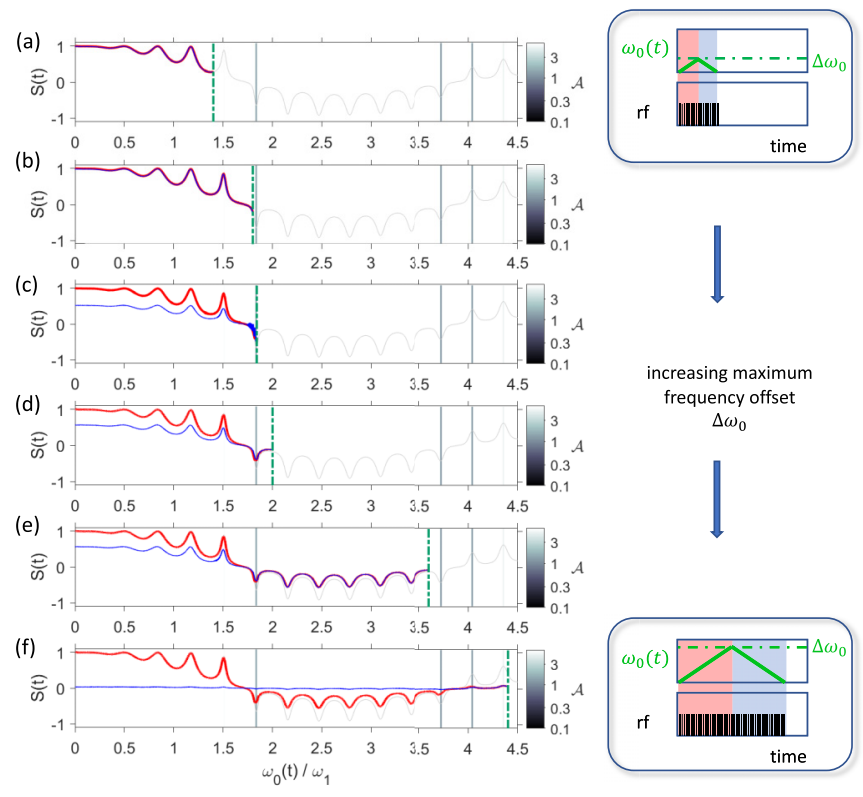

FIG. 4. Measured in-phase echo amplitudes versus instantaneous normalized offset frequency $\omega_{0}(t) / \omega_{1}$ for a CPMG sequence with a bilinear $B_{0}$ ramp. As indicated schematically on the right panels, the field was linearly ramped up from on resonance to a value that corresponded to a maximum frequency offset of $\Delta \omega_{0}$ indicated by the green dash-dotted lines. The up ramp was immediately followed by a ramp down back to on resonance. The signals for the up ramp, $S_{\uparrow}$, and the down ramp, $S_{\downarrow}$, are shown in red and in blue, respectively. The magnitude of the normalized ramp rates $d \tilde{\omega}_{0} / d \tau$ for both up and down ramps was fixed at $1 \times 10^{-3}$ and the echo spacing was set at $t_{E} / t_{180}=6.4$. The light gray curve shows the expected signal for a purely adiabatic behavior [Eq. (B1)] with $a_{C P M G}=1$. The vertical dark lines show the nonadiabatic regions, based on the calculated values of $\mathcal{A}$ displayed in grayscale. From (a) to (f), the value of $\Delta \omega_{0}$ was systematically increased from around $1.4 \omega_{1}$ to $4.4 \omega_{1}$, as indicated by the green dash-dotted lines.

field variations up to $\Delta \omega_{0}=1.8 \omega_{1}$ shown in Fig. 4(b). During this field sweep, the transverse magnetization temporarily vanishes and becomes negative, all in a fully reversible manner.

However, as soon as $\Delta \omega_{0}$ exceeds the critical value of $1.83 \omega_{1}$ and the spin dynamics enters the nonadiabatic region, the signal fails to fully refocus when the offset field returns to zero. The response during the ramp-down $S_{\downarrow}\left(\omega_{0}\right)$ is generally rescaled from the response during the ramp-up $S_{\uparrow}\left(\omega_{0}\right)$. The scaling factor can be interpreted as the amplitude $a_{C P M G}$ of the CPMG mode in Eq. (B1). It is a function of the number of nonadiabatic regions encountered during the field sweep. For frequency offsets between the last nonadiabatic region and $\Delta \omega_{0}$, the response is again reversible.

In Fig. 5 we plot the signal that is acquired at the end of the scan (when the field has returned to its original value) $S_{R T O}$ versus the maximum frequency offset during the sweep $\Delta \omega_{0}$. This can be interpreted as the amplitude $a_{C P M G}$ of the CPMG mode at the end of the scan. The results shown in Fig. 5 exhibit a simple steplike function. The locations of the steps coincide exactly with the predicted nonadiabatic regions where $\mathcal{A}$ approaches or becomes less than 1 . 

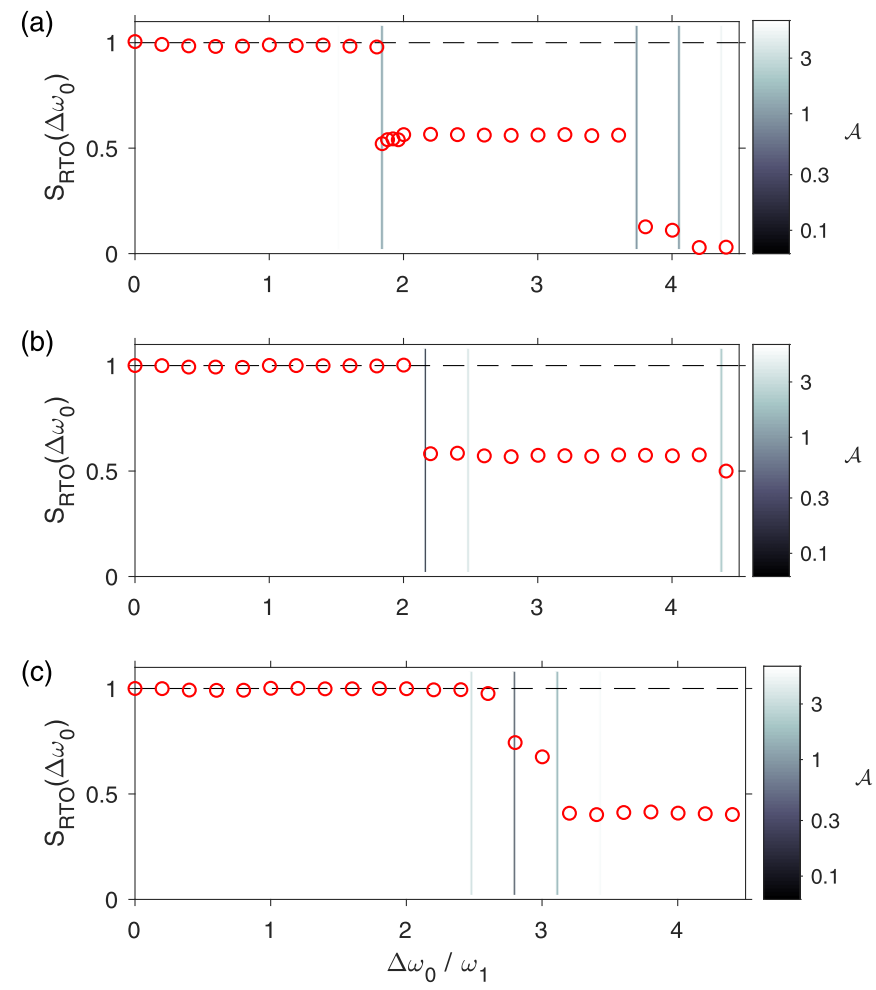

FIG. 5. Measured in-phase signal at the end of the scan $S_{R T O}$ versus the maximum frequency offset during the sweep $\Delta \omega_{0}$ for the bilinear field ramps shown in Fig. 4. The duration of the refocusing pulse $t_{p}$ was set to (a) $\frac{\pi}{\omega_{1}}$, (b) $\frac{5 \pi}{6 \omega_{1}}$, and (c) $\frac{2 \pi}{3 \omega_{1}}$, corresponding to nominal $180^{\circ}, 150^{\circ}$, and $120^{\circ}$ pulses, respectively. The vertical dark lines show the nonadiabatic regions, based on the calculated values of $\mathcal{A}$ displayed in grayscale.

The experimental results of Fig. 5 show a striking simplicity. The degree of refocusing at the end of the scan $S_{R T O}$ depends only on the number of adiabatic regions that were crossed during the scan. It does not depend on the exact value of $\Delta \omega_{0}$ within a particular adiabatic region. These measurements therefore allow a direct identification of the adiabatic and nonadiabatic regions.

Based on numerical simulations with uniform fields reported in [7], this simplicity was not anticipated. As the applied field ramps through a nonadiabatic region, some magnetization is converted from the CPMG to the CP modes. In the following adiabatic regime, the $\mathrm{CP}$ magnetization propagates with nonunity eigenvalues and acquires nonzero dynamical and possibly geometric phases that are sensitive to $\Delta \omega_{0}$. After the field is reversed and crosses again the nonadiabatic region, part of the remaining CPMG magnetization will again be converted to the CP mode. In addition, some of the $\mathrm{CP}$ magnetization is converted back to the CPMG mode and is added to the CPMG mode. We expect that the contribution of the CPMG mode converted from the CP mode depends sensitively on $\Delta \omega_{0}$ due to the variation of the accumulated phase. The absence of a dependence of $S_{R T O}$ on $\Delta \omega_{0}$ within an adiabatic region in the experimental results of Fig. 5 indicates that the converted CP magnetization does not make any net contribution to the detected signal $S_{R T O}$.
The absence of sensitivity of $S_{R T O}$ on $\Delta \omega_{0}$ can be understood in our experiments as follows. As hinted in previous discussions, the process of driving the magnetization (spin) through a nonadiabatic region can be partitioned into three stages within the adiabatic impulse approximation [19]: (1) the adiabatic evolution before the transition, (2) the nonadiabatic (impulse) transition, and (3) the adiabatic evolution after the transition. Similar to the Landau-Zener transition, the transition in the nonadiabatic region can be organized into a unitary matrix describing the transition rates between different states. With the small relative field inhomogeneity $6.7 \times 10^{-3}$, spins subjected to different field strengths essentially follow the same transition matrix in our experiments [1]. In particular, because the nonadiabatic transition occurs in a small range of $\omega_{0} / \omega_{1}$, no strong phase variations are expected due to the field inhomogeneity in this region. In contrast, the adiabatic evolution of the magnetization for the $\mathrm{CP}$ mode is strongly affected by the inhomogeneity of the applied bias field (cf. Appendix C), which in general induces a phase spreading of the CP components for spins subjected to different field strengths and hence leads to the dephasing, i.e., shorter $T_{2}^{*}$, for the CP components. As shown in Appendix C, except at some specific offset frequency $\omega_{0}$ where the phase spreading of $\mathrm{CP}$ modes reverts due to the dispersion of the $\mathrm{CP}$ mode phase spectrum, the magnetization of CP modes is effectively diminished due to this phase spreading even for the small relative field inhomogeneity in our experimental setup. In addition to the field inhomogeneity effect, the $\mathrm{CP}$ components are also preferentially attenuated by diffusion effects.

Now, when an ensemble of CP modes with randomized phases is driven through the nonadiabatic region, the CP modes converted back to CPMG modes will still retain these randomized phases. As a result, the ensemble-averaged CPMG signals converted from these CP modes become negligible, which leads to the $\Delta \omega_{0}$-independent $S_{R T O}$ signal. This occurs as long as the accumulated phases of these $\mathrm{CP}$ modes are well randomized before entering the nonadiabatic region. On the other hand, some of the CPMG modes will still be converted to $\mathrm{CP}$ modes, which will quickly dephase due to the field inhomogeneity. This implies that the magnitude of $S_{R T O}\left(\Delta \omega_{0}\right)$ should decrease monotonically with every additional nonadiabatic event, as is indeed observed in our experiments.

\section{Measurements with modified refocusing cycles}

The response in time-dependent fields can be modified by replacing the standard $180^{\circ}$ refocusing pulses with composite or phase-modulated pulses [20,21]. It is possible to make the system less or more sensitive towards field fluctuations. Such optimization can be considered a form of Hamiltonian engineering. The present analysis shows that the key quantity to optimize is the critical velocity $v_{0 \text {,crit }}\left(\omega_{0}\right)$, which in turn is determined by the modal properties of a single refocusing cycle.

To find sequences more robust towards field fluctuations, optimal control based algorithms [21-23] can be used to systematically search for refocusing cycles with modal structures that have no near degeneracies and associated low critical velocities for the range of offset frequencies of interest. This 

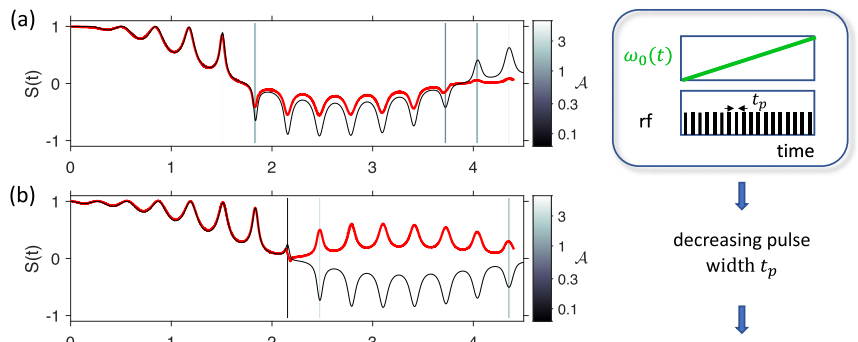

decreasing pulse width $t_{p}$

$\Downarrow$
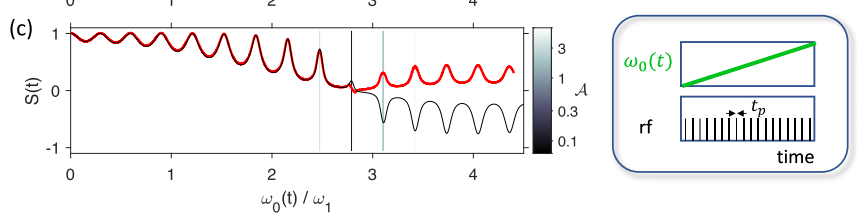

FIG. 6. Measured in-phase (red) echo amplitudes for a CPMG sequence with a linearly increasing $B_{0}$ field, starting on resonance for different refocusing pulses. The duration of the refocusing pulse $t_{p}$ was set to (a) $\frac{\pi}{\omega_{1}}$, (b) $\frac{5 \pi}{6 \omega_{1}}$, and (c) $\frac{2 \pi}{3 \omega_{1}}$, corresponding to nominal $180^{\circ}, 150^{\circ}$, and $120^{\circ}$ pulses, respectively. The normalized ramp rate $d \tilde{\omega}_{0} / d \tau$ was fixed at $1 \times 10^{-3}$ and the echo spacing at $t_{E} / t_{180}=6.4$. The signal was normalized by a standard CPMG signal to account for relaxation. The black lines show the theoretical expectation for the fully adiabatic case based on Eq. (B1). The vertical dark lines show the nonadiabatic regions, based on the calculated values of $\mathcal{A}$ displayed in grayscale. The panels on the right give a schematic indication of the experimental procedure.

will eliminate nonadiabatic regions and result in a fully adiabatic spin dynamics. It could also be desirable to minimize the signal modulation with variable offset frequency. This could be accomplished by searching for refocusing cycles that maximize the critical velocities while simultaneously minimizing the variability in the direction of the eigenvector of the CPMG mode versus $\omega_{0}$.

As a simple illustration of this general approach of modified CPMG sequences, we present in Fig. 6 measurements with shortened refocusing pulses. The pulse durations were reduced from the default value of $t_{180}=\pi / \omega_{1}$ by $16 \%$ or $33 \%$ without increasing the pulse amplitude. The pulses now correspond to nominal $150^{\circ}$ or $120^{\circ}$ pulses, respectively. The analytical expressions of the modal properties of the propagator in Appendix A show that a reduction of the pulse durations moves the location of the nonadiabatic regions to higher offset frequencies, thus increasing the range of the first adiabatic region. This prediction is confirmed by the experimental measurements of Fig. 6: The echo amplitudes with the shortened refocusing pulses follow the adiabatic result to a significantly larger offset frequency. The modified sequences have an improved robustness towards the amplitude of field fluctuations that can be fully refocused. As shown in Fig. 5, with $120^{\circ}$ refocusing pulses field fluctuations up to about $2.8 B_{1}$ can be fully recovered without loss of signal, while with standard $180^{\circ}$ refocusing pulses this can only be achieved with fluctuations up to about $1.8 B_{1}$. However, this increased adiabatic range is associated with a more pronounced modulation of the echo amplitudes at small offset frequencies. It might be possible to find more complex refocusing pulses that can eliminate this drawback of enhanced signal modulation but retain the increased adiabatic range.

\section{CONCLUSION}

We have applied and tested a framework to characterize the response of CPMG-like sequences to time-dependent magnetic fields. It is based on an effective Hamiltonian approach that allows the decomposition of the magnetization in terms of the eigenmodes of the propagator. We have shown that the response can be generally classified into adiabatic behavior that is occasionally interrupted by nonadiabatic events.

In the adiabatic regime, the simple analytical expressions have been experimentally confirmed. This can be viewed as a test of the structure of the effective Hamiltonian. In the presence of moderate field inhomogeneities, the magnetization associated with the $\mathrm{CP}$ modes generally dephases quickly. The detected magnetization is then dominated by the magnetization of the CPMG eigenmode that is effectively spin locked to its eigenvector and robust towards field inhomogeneities. Changes in the occupation of the eigenmodes require a nonadiabatic event. They occur in narrow ranges of offset frequencies where the adiabaticity condition $\mathcal{A} \gg 1$ is not fulfilled. The adiabaticity parameter $\mathcal{A}$ is the ratio of an intrinsic offset-dependent critical velocity that is derivable from modal properties of the propagator to the instantaneous ramp rate of the applied field.

In this study we have tested the theory using piecewise linear field variations. We expect the treatment to hold for more general field fluctuations, including random field variations. As long as the instantaneous adiabaticity parameter $\mathcal{A}(t)$ [determined by the instantaneous values of normalized Larmor frequency offset $\tilde{\omega}_{0}(t)$ and its rate of change $\left.d \tilde{\omega}_{0}(t) / d \tau\right]$ is much larger than 1, the spin dynamics is adiabatic and the simple adiabatic expressions describe the response.

This effective Hamiltonian approach applies more generally to any CPMG-like sequence that consists of an initial excitation pulse followed by identical refocusing cycles. The refocusing cycle not only can consist of a single rf pulse, but can contain any composite pulse, a combination of $\mathrm{rf}$ pulses, or frequency sweeps. Thus, it is possible to optimize the response to time-dependent magnetic fields by finding refocusing cycles with the desired properties of the critical velocity and other modal properties. Sequences that are more robust towards temporal field variations are associated with high critical velocities without any pronounced minima in the relevant range of offset frequencies. Alternatively, it might be desirable to find sequences that show an enhanced sensitivity to field fluctuations. In that case, the goal is to use an operating point with a low critical velocity, near a singular point where the different modes become degenerate and modal transitions occur even with small field fluctuations.

From the reversibility test with the bilinear field ramps to a maximum offset frequency $\Delta \omega_{0}$ we observe a rather simple steplike structure of the returning CPMG signals with respect to the number of nonadiabatic events encountered. We argue that this is due to the absence of CP mode contributions in an inhomogeneous applied field environment. This surprisingly simple response indicates the robustness for the measured CPMG magnetization. On the other hand, it also hinders the observation of the interference effects between two or more nonadiabatic transition events [7]. By carefully inspecting the in- and out-of phase signals during the ramping 
process, we observe the refocusing of the $\mathrm{CP}$ mode signals at specific detuning frequencies (cf. Appendix C) with the mechanism similar to that for the gradient echo. Hence, with the reduction of the field inhomogeneity, we are hopeful that the interference effects can be observed. Coupled with Hamiltonian engineering through the composite pulse sequence, our system thus has the potential to become a simulator to probe dynamical responses and interference phenomena similar to certain quantum systems [1-3].

\section{APPENDIX A: PROPERTIES OF EIGENMODES OF THE REFOCUSING CYCLE WITH A RECTANGULAR RF PULSE}

We consider a refocusing cycle of duration $t_{E}$ that contains a single rf pulse of duration $t_{p}$ centered in the middle of the cycle. The rf pulse is linearly polarized with a carrier frequency of $\omega_{r f}$ and an amplitude $B_{1, \perp}$, resulting in a nominal nutation frequency of $\omega_{1} \equiv \gamma B_{1, \perp} / 2$. The static field is $B_{0}$, resulting in an offset between the Larmor frequency and applied rf of $\omega_{0}(t) \equiv \gamma B_{0}(t)-\omega_{r f}$. For independent spins $1 / 2$, the echo-to-echo evolution can then be described using average Hamiltonian theory by an effective magnetic field $\gamma \vec{B}_{\text {eff }}=$ $\alpha / t_{E} \hat{n}$, where $\gamma$ is the gyromagnetic ratio. For a rectangular rf pulse, the direction $\hat{n}$ and amplitude $\alpha$ of this resulting effective magnetic field can be obtained from the expressions

$$
\begin{gathered}
n_{\perp}=\frac{1}{\Delta} \frac{\omega_{1}}{\Omega} \sin \beta_{2}, \\
n_{z}=\frac{1}{\Delta}\left(\sin \beta_{1} \cos \beta_{2}+\frac{\omega_{0}}{\Omega} \cos \beta_{1} \sin \beta_{2}\right), \\
\cos \left(\frac{\alpha}{2}\right)=\cos \beta_{1} \cos \beta_{2}-\frac{\omega_{0}}{\Omega} \sin \beta_{1} \sin \beta_{2},
\end{gathered}
$$

where

$$
\begin{gathered}
\Omega=\sqrt{\omega_{0}^{2}+\omega_{1}^{2}}, \\
\beta_{1}=\omega_{0}\left(t_{E}-t_{p}\right) / 2 \\
\beta_{2}=\Omega t_{p} / 2 \\
\Delta^{2}=\left(\frac{\omega_{1}}{\Omega} \sin \beta_{2}\right)^{2}+\left(\sin \beta_{1} \cos \beta_{2}+\frac{\omega_{0}}{\Omega} \cos \beta_{1} \sin \beta_{2}\right)^{2} .
\end{gathered}
$$

\section{APPENDIX B: ANALYTICAL RESULTS FOR THE ADIABATIC REGIME}

In the presence of field inhomogeneities, the detected signal is dominated by the contribution of the CPMG mode. In the fully adiabatic limit of vanishing small ramp rates $d \tilde{\omega}_{0} / d \tau$, the magnetization of the CPMG mode is spin locked to the eigenvector $\hat{n}$ derived from the static case. To lowest order in the ramp rate, the signal is given by

$$
S(t)=a_{C P M G} n_{\perp}(t) .
$$

Here $a_{C P M G}$ is the amplitude or occupation of the CPMG mode and $n_{\perp}(t)$ is the transverse component of $\hat{n}$ [Eq. (A1)] at the instantaneous offset frequency $\omega_{0}(t)$. In this limit, the signal is purely in phase with the refocusing pulses.
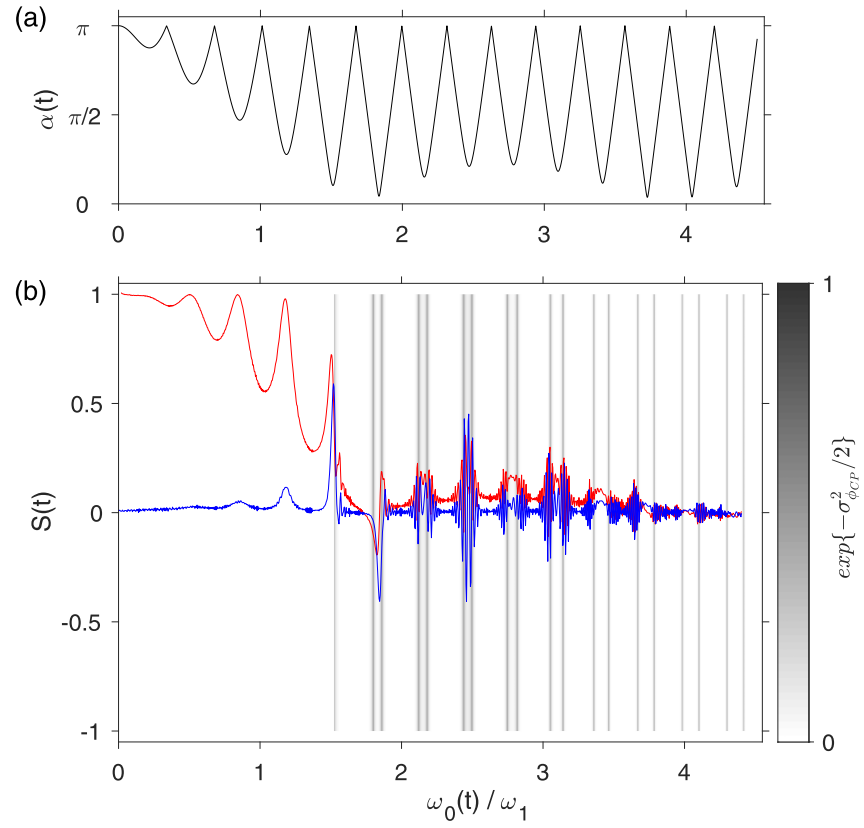

FIG. 7. Experimental demonstration of gradient-echo-like signals generated by the $\mathrm{CP}$ contributions. (a) Oscillatory nature of the instantaneous values of $\alpha$ versus the normalized offset frequency $\tilde{\omega}_{0}(t)=\omega_{0}(t) / \omega_{1}$, calculated from Eq. (A3). (b) The red and blue lines show, respectively, the experimental in-phase and out-of-phase signals detected during a linear ramp versus $\tilde{\omega}_{0}(t)$ with $d \tilde{\omega}_{0} / d \tau=$ $4 \times 10^{-3}$ and $t_{E} / t_{180}=6.4$. The grayscale shows the simple prediction of $\exp \left\{-\sigma_{\phi_{C P}}^{2} / 2\right\}$. This can be interpreted as the visibility of gradient echoes, as discussed in the text.

For finite ramp rates, the eigenvector for the CPMG eigenmode acquires a small out-of-phase component. A perturbation calculation to first order in $1 / \mathcal{A}$ yields

$$
\begin{aligned}
S_{x}^{(1)}(t) & =\frac{a_{C P M G}}{\sqrt{1+1 / \mathcal{A}(t)^{2}}}\left[\cos (\delta \epsilon) n_{\perp}(t)-1 / \mathcal{A}(t) \sin (\delta \epsilon)\right], \\
\left|S_{y}^{(1)}(t)\right| & =\frac{a_{C P M G}}{\sqrt{1+1 / \mathcal{A}(t)^{2}}}\left[\sin (\delta \epsilon) n_{\perp}(t)+1 / \mathcal{A}(t) \cos (\delta \epsilon)\right] .
\end{aligned}
$$

Here $\delta \epsilon \equiv \frac{t_{E}^{2}}{8} \frac{d \omega_{0}}{d t}$

\section{APPENDIX C: EFFECT OF FIELD INHOMOGENEITIES ON THE SIGNAL GENERATED BY THE CP MODES}

In the continuous limit, the dynamic phase of the CP mode in the adiabatic regime for a linear field ramp is given by

$$
\phi_{C P}\left(\tilde{\omega}_{0}\right)=\left(\frac{d \tilde{\omega}_{0}}{d \tau}\right)^{-1} \int_{\tilde{\omega}_{0, \text { start }}}^{\tilde{\omega}_{0}} \alpha\left(\tilde{\omega}_{0}^{\prime}\right) d \tilde{\omega}_{0}^{\prime} .
$$

Here $\tilde{\omega}_{0 \text {,start }}$ is the normalized offset frequency where the $\mathrm{CP}$ mode has been initialized. When the applied field $\omega_{0}$ is nonuniform across the sample, the phase of the CP component is also nonuniform. This leads to a $T_{2}^{*}$-like decay of the detected signal of the $\mathrm{CP}$ component $S_{C P}$. Assuming that the inhomogeneities of the applied field is characterized by a Gaussian distribution with a standard deviation $\sigma_{\omega_{0}}$, the resulting distribution of the phases has a standard deviation 
that is to first order given by

$$
\sigma_{\phi_{C P}}^{2}=\sigma_{\omega_{0}}^{2}\left(\frac{d \tilde{\omega}_{0}}{d \tau}\right)^{-2}\left(\int_{\tilde{\omega}_{0, \mathrm{start}}}^{\tilde{\omega}_{0}} \tilde{\omega}_{0}^{\prime} \frac{d \alpha\left(\tilde{\omega}_{0}^{\prime}\right)}{d \tilde{\omega}_{0}^{\prime}} d \tilde{\omega}_{0}^{\prime}\right)^{2} .
$$

The field inhomogeneity reduces the detected CP signal by $\exp \left\{-\sigma_{\phi_{C P}}^{2} / 2\right\}$ compared to the case of uniform $\omega_{0}$. In the experimental results presented above, the condition $\sigma_{\phi_{C P}}^{2}\left(\tilde{\omega}_{0}\right) \gg$ 1 is generally well fulfilled in the adiabatic regime except in the near vicinity of the nonadiabatic regimes. Consequently, the detected signals are dominated by the CPMG modes and the $\mathrm{CP}$ modes make no significant contributions.

However, there are conditions when $\sigma_{\phi_{C P}}^{2}$ becomes small or even vanishes, even in the presence of field inhomogeneities. In such cases, signals from the CP modes are detected. This is made possible by the oscillatory nature of $d \alpha / d \tilde{\omega}_{0}$ with $\tilde{\omega}_{0}$. At particular offset frequencies, the integral in Eq. (C2) becomes zero. At these special points, the overall phase of the
$\mathrm{CP}$ contribution is to first order independent of the field inhomogeneity (but in general nonzero) and the $\mathrm{CP}$ modes form gradient-echo-like signals. Given the $\left(\frac{d \tilde{\omega}_{0}}{d \tau}\right)^{-2}$ dependence of $\sigma_{\phi_{C P}}^{2}$, such gradient echoes are more pronounced at higher ramp rates.

An example of such CP gradient echoes are shown in the experimental results of Fig. 7. These data were acquired at a ramp rate $d \tilde{\omega}_{0} / d \tau=4 \times 10^{-3}$ and $t_{E} / t_{180}=6.4$. At distinct offset frequencies, oscillating out-of-phase signals show the formation of CP gradient echoes. The position of these echoes corresponds well to the locations where $\exp \left\{-\sigma_{\phi_{C P}}^{2} / 2\right\}$ approaches 1 . Here $\sigma_{\phi_{C P}}$ was calculated from Eq. (C2) using $\tilde{\omega}_{0 \text {,start }}$ as the end of the first nonadiabatic regime encountered. This calculation is a simplification. It implicitly assumes that only the first nonadiabatic event generates $\mathrm{CP}$ contributions and that the phase shifts induced by the subsequent nonadiabatic regions can be ignored. Despite this approximation, it gives a good qualitative indication where $\mathrm{CP}$ gradient echoes can form.
[1] S. Shevchenko, S. Ashhab, and F. Nori, Landau-ZenerStückelberg interferometry, Phys. Rep. 492, 1 (2010).

[2] S. Gustavsson, J. Bylander, and W. D. Oliver, Time-Reversal Symmetry and Universal Conductance Fluctuations in a Driven Two-Level System, Phys. Rev. Lett. 110, 016603 (2013).

[3] A. Ferrón, D. Domínguez, and M. J. Sánchez, Mesoscopic fluctuations in biharmonically driven flux qubits, Phys. Rev. B 95, 045412 (2017).

[4] C. S. E. van Ditzhuijzen, A. Tauschinsky, and H. B. van Linden van den Heuvell, Observation of Stückelberg oscillations in dipole-dipole interactions, Phys. Rev. A 80, 063407 (2009).

[5] A. Shytov, D. Ivanov, and M. Feigel'man, Landau-Zener interferometry for qubits, Eur. Phys. J. B 36, 263 (2003).

[6] M. Sillanpää, T. Lehtinen, A. Paila, Y. Makhlin, and P. Hakonen, Continuous-Time Monitoring of Landau-Zener Interference in a Cooper-Pair Box, Phys. Rev. Lett. 96, 187002 (2006).

[7] M. D. Hürlimann, S. Utsuzawa, and C.-Y. Hou, Spin Dynamics of the Carr-Purcell-Meiboom-Gill Sequence in time-dependent Magnetic Fields, Phys. Rev. Appl. 12, 044061 (2019).

[8] H. Y. Carr and E. M. Purcell, Effects of diffusion on free precession in nuclear magnetic resonance experiments, Phys. Rev. 94, 630 (1954).

[9] S. Meiboom and D. Gill, Modified spin-echo method for measuring nuclear relaxation times, Rev. Sci. Instrum. 29, 688 (1958).

[10] W. A. Stewart, A. L. Mackay, K. P. Whittall, G. R. W. Moore, and D. W. Paty, Spin-spin relaxation in experimental allergic encephalomyelitis. analysis of CPMG data using a non-linear least squares method and linear inverse theory, Magn. Reson. Med. 29, 767 (1993).

[11] J. P. Loria, M. Rance, and A. G. Palmer, A relaxationcompensated Carr-Purcell-Meiboom-Gill sequence for characterizing chemical exchange by NMR spectroscopy, J. Am. Chem. Soc. 121, 2331 (1999).

[12] K. Lim, T. Nguyen, T. Mazur, D. E. Wemmer, and A. Pines, Sensitivity enhancement in multiple-quantum NMR experiments with CPMG detection, J. Magn. Reson. 157, 160 (2002).
[13] L. Viola and S. Lloyd, Dynamical suppression of decoherence in two-state quantum systems, Phys. Rev. A 58, 2733 (1998).

[14] P. Cappellaro, J. S. Hodges, T. F. Havel, and D. G. Cory, Principles of control for decoherence-free subsystems, J. Chem. Phys. 125, 044514 (2006).

[15] M. J. Biercuk, H. Uys, A. P. VanDevender, N. Shiga, W. M. Itano, and J. J. Bollinger, Optimized dynamical decoupling in a model quantum memory, Nature (London) 458, 996 (2009).

[16] M. D. Hürlimann and D. D. Griffin, Spin dynamics of Carr-Purcell-Meiboom-Gill-like sequences in grossly inhomogeneous $B_{0}$ and $B_{1}$ fields and application to NMR well logging, J. Magn. Reson. 143, 120 (2000).

[17] M. D. Hürlimann and N. J. Heaton, in Mobile NMR and MRI: Developments and Applications, edited by M. L. Johns, E. O. Fridjonsson, S. J. Vogt, and A. Haber (Royal Society of Chemistry, Cambridge, 2015), pp. 11-85.

[18] V. D. M. Koroleva, S. Mandal, Y. Q. Song, and M. D. Hürlimann, Broadband CPMG sequence with short composite refocusing pulses, J. Magn. Reson. 230, 64 (2013).

[19] B. Damski, The Simplest Quantum Model Supporting the Kibble-Zurek Mechanism of Topological Defect Production: Landau-Zener Transitions from A New Perspective, Phys. Rev. Lett. 95, 035701 (2005).

[20] M. D. Hürlimann, Carr-Purcell sequences with composite pulses, J. Magn. Reson. 152, 109 (2001).

[21] T. W. Borneman, M. D. Hürlimann, and D. G. Cory, Application of optimal control to CPMG refocusing pulse design, J. Magn. Reson. 207, 220 (2010).

[22] N. Khaneja, T. Reiss, C. Kehlet, T. Schulte-Herbrüggen, and S. J. Glaser, Optimal control of coupled spin dynamics: Design of NMR pulse sequences by gradient ascent algorithms, J. Magn. Reson. 172, 296 (2005).

[23] S. Mandal, T. W. Borneman, V. D. M. Koroleva, and M. D. Hürlimann, Direct optimization of signal-to-noise ratio of CPMG-like sequences in inhomogeneous fields, J. Magn. Reson. 247, 54 (2014). 